'ResplRAChile, Santiago, Chile. ${ }^{2}$ Instituto de Salud Pública Andrés Bello, Universidad Andrés Bello. Santiago, Chile. ${ }^{3}$ Postgrados. Facultas de Ciencias de la Rehabilitación. Universidad Andrés Bello. Santiago, Chile. ${ }^{4}$ Departamento de Salud Pública y Epidemiología. Universidad de Santiago de Chile Santiago, Chile.

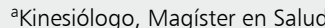
Pública (MPH).

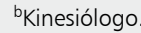

Recibido el 18 de julio de 2014, aceptado el 1 de diciembre de 2014.

Correspondencia a: MPH Claudio Olmos G. claudioolmos@gmail.com

\section{Epidemiología de las consultas respiratorias de adultos en Santiago de Chile desde 2003 a 2008}

\author{
CLAUDIO OLMOS ${ }^{1,2,3, \mathrm{a}}$, PEDRO MANCILLA ${ }^{1,3, \mathrm{~b}}$, \\ LUIS MARTÍNEZ ${ }^{4}$, PEDRO ASTUDILLO ${ }^{1,3}$
}

\section{Primary care consultations due to respiratory diseases in the period 2003-2008}

Background: Respiratory diseases are the third cause of death, and the second cause of hospitalization among people aged 65 years or more in Chile. Aim: To analyze the distribution of consultations due to respiratory diseases among adults living in Metropolitan Santiago. Material and Methods: A daily registry of all consultations of patients older than 15 years old in seven public primary care centers, was carried out between January 2003 and December 2008. Consultations were classified as having non-respiratory or respiratory causes. The latter were broke down in upper or lower respiratory diseases, pneumonia, chronic obstructive pulmonary disease (COPD) and asthma. Results: A total of $1,170,941$ consultations were registered and $19 \%$ were due to respiratory diseases. Of these, $46 \%$ were due to upper respiratory diseases, $31 \%$ due to lower respiratory diseases, $8 \%$ due to COPD, $6 \%$ due to pneumonia, $5 \%$ due to asthma and $4 \%$ due to other respiratory causes. Pneumonia and COPD were more frequent among consultants older than 65 years. Conclusions: Consultations due to respiratory diseases are approximately one fifth of all primary care consultations. Older people often have more chronic and severe diseases.

(Rev Med Chile 2015; 143: 30-38)

Key words: Adult; Epidemiology; Morbidity; Respiratory tract diseases.
E n Chile, las enfermedades del sistema respiratorio constituyen el tercer grupo de causas de muerte, sólo superado por enfermedades del sistema circulatorio y tumores ${ }^{1}$, también son la segunda causa de egreso hospitalario en mayores de 65 años ${ }^{2}$.

Pese a la importancia que tienen en Chile las enfermedades respiratorias en mortalidad y hospitalizaciones, hay escasa evidencia científica respecto de la proporción de ellas dentro de las consultas de morbilidad del adulto (CA), pues los estudios existentes se limitan a un sólo centro de salud $d^{3-8}$ o no son actuales ${ }^{9}$. Asimismo, dichos estudios no otorgan información precisa respecto al comportamiento estacional de las consultas por causa respiratoria en los últimos años. Conocer esta información es relevante, si se considera el envejecimiento que ha experimentado la población chilena ${ }^{10}$ y el consecuente aumento de la demanda por prestaciones de salud en determinadas épocas del año.

Con estas limitaciones, para las consultas de adultos por causa respiratoria se han descrito en la literatura cifras de promedio anual que van desde $10 \%$ hasta $16 \%$ del total de $\mathrm{CA}^{9,11}$.

Por otro lado, es razonable pensar que la distribución de las consultas no debe ser la misma en las distintas épocas del año, ni en las distintas etapas de la edad adulta. Además, la oferta de atención por parte del sistema público de salud tiene limitaciones, que se hacen más notorias precisamente en los períodos de alta demanda 
asistencial, donde concurren en forma simultánea factores de riesgo como las infecciones virales, el frío y la contaminación aérea.

Todo eso supone un desafío para las autoridades, en cuanto a tener una información más sensible respecto de lo que pasa con la morbilidad del adulto en los centros correspondientes a la Atención Primaria de Salud (APS). Es por esto que el presente trabajo se propone describir la ocurrencia y distribución de las consultas de morbilidad de adultos, esencialmente las de carácter respiratorio, en sujetos mayores de 15 años, con el objetivo de dar a conocer su composición diagnóstica, evolución estacional, y a lo largo del período, y sus variaciones según grupo etario, como elementos indispensables para poder replantear estrategias, focalizar recursos y tomar decisiones adecuadas.

El presente estudio continúa una línea de investigación llevada a cabo por los autores, y que se ha publicado recientemente en la Revista Panamericana de Salud Pública ${ }^{12}$.

\section{Materiales y Métodos}

La presente investigación obedece a un diseño descriptivo, con información recogida prospectivamente durante un período comprendido entre el 1 de enero de 2003 y el 31 de diciembre de 2008. Fueron seleccionados 7 centros de salud de APS, representativos de los 4 puntos cardinales de la Provincia de Santiago, mediante la metodología de
Centros Centinela, propuesta por UNICEF ${ }^{14}$. Estos centros fueron: "Avendaño" de la comuna de Lo Prado, "Centro de Salud No 5" de Santiago, "Dr. Anibal Ariztía" de Las Condes, "Joao Goulart" de La Granja, "La Feria" de Pedro Aguirre Cerda, "Lo Barnechea" de la comuna de Lo Barnechea y "Villa O'Higgins" de La Florida (Tabla 1). La población asignada a los 7 Centros Centinelas no presentó variaciones significativas durante el período del estudio.

En todos los centros elegidos, funcionaba una "Sala ERA"11, unidad operativa del Programa Nacional de Enfermedades Respiratorias del Adulto de Chile, instaladas desde la puesta en marcha del Programa, en el año 2001.

Para llevar a cabo esta investigación, por cada Centro se contó con el kinesiólogo encargado de la respectiva sala, a lo menos un médico y una o dos personas del área administrativa. Dicho equipo estuvo a cargo de efectuar los registros diarios y enviarlos al equipo de investigadores del nivel central, para su posterior consolidación y análisis. Con el objetivo de minimizar sesgos de información, desde el nivel central, un equipo de tres profesionales, además de personal administrativo, se mantuvo en contacto periódico con los funcionarios instalados en los centros, con el fin de informar, capacitar y supervisar su labor, asegurando la calidad de los registros, de las comunicaciones y el exacto ingreso de la información a un sistema de base de datos computacional.

Tabla 1. Ubicación de centros centinela (CC), por comuna, servicio de salud y población inscrita o asignada, provincia de Santiago, Chile

\begin{tabular}{|lllc|}
\hline Establecimiento & Comuna & Servicio Metropolitano & Población \\
Avendaño & Lo Prado & Occidente & 30.019 \\
Centro de Salud N ${ }^{\circ} 5$ & Santiago & Central & 45.597 \\
Dr. Aníbal Ariztía & Las Condes & Oriente & 43.312 \\
João Goulart & La Granja & Suroriente & 31.960 \\
La Feria & Pedro Aguirre Cerda & Sur & 28.869 \\
Lo Barnechea & Lo Barnechea & Oriente & 36.153 \\
Villa O'Higgins & La Florida & Suroriente & 39.482 \\
Población total en CC & & & 255.392 \\
\hline Población total en provincia de Santiago & & & $4.311 .133^{*}$ \\
Representación de los CC (\%) & & & 5,9 \\
\hline
\end{tabular}

*Población correspondiente al Censo 2002. Fuente: elaboración de los autores. 
Para disponer de información estandarizada y periódica, se elaboraron un formulario para la recolección y transmisión de la información diaria y resumida de los diagnósticos, y un instructivo para la clasificación de las enfermedades respiratorias, que tuvo por objeto interpretar la forma cómo efectúan el diagnóstico los médicos y convertirla en datos que pudieran incorporarse al formulario. Mediante estos instrumentos, se recolectó prospectivamente la información diaria de todas las consultas de mayores de 15 años, especificando el diagnóstico cuando éste era de tipo respiratorio. Así, las CA se agruparon en: consultas por causa no respiratoria (CNR) y consultas por causa respiratoria (CR). A su vez, estas últimas se desglosaron en: consultas por enfermedades agudas de vía aérea alta (ERAa), enfermedades agudas de vía aérea baja (ERAb), neumonía, enfermedad pulmonar obstructiva crónica (EPOC) y asma.

Es relevante destacar que sólo fueron consideradas las consultas de morbilidad, y no se estableció un sistema para diferenciar la primera consulta correspondiente a cada episodio de enfermedad; es decir, se trata consultas y no de casos de enfermedad. Tampoco hay diferenciación de las CNR. Todas las anteriores fueron estratificadas, además, por grupo etario ( 15 a 44 años, 45 a 64 años y 65 y más años).

El envío de formularios se realizó diariamente, vía fax o correo electrónico, lo que permitió obtener los registros con un retraso no mayor de 24 horas desde que la información fue generada, y a partir de ellos, construir una planilla de datos electrónicos.

Los resultados se expresan como proporción del total de consultas, y no en tasas por población, debido a que el número total diario de consultas en APS tiene un límite que está dado por la cantidad de horas médicas disponibles para la atención y, por lo tanto, no es extensible a la demanda.

Una vez procesados los datos obtenidos, fueron agrupados mensualmente y expresados en función de máximos, mínimos y medianas, lo cual permite la construcción de canales endémicos, que puedan orientar al lector respecto de las características de un año "normal" de consultas. Con el objeto de evitar sesgos producto de un posible año epidémico, se procedió a excluir los valores máximos alcanzados durante el período, para esta causa. En consecuencia, los máximos considerados para la elaboración de los canales endémicos, correspon- den al segundo valor máximo de cada mes.

Para el análisis estadístico, se ajustó un modelo de regresión lineal utilizando como variable dependiente a la proporción de consultas por diagnóstico específico, y como variable independiente, el tiempo, así como la prueba de ANOVA, con contraste de Scheffé, para comparar diferencias entre los grupos etarios según diagnóstico.

\section{Resultados}

\section{Resultados generales}

Desde el 1 de enero de 2003, hasta el 31 de diciembre de 2008, se registró un total de 1.170.941 consultas en mayores de 15 años. De ellas, 223.617 $(19,10 \%)$ fueron por causa respiratoria. La distribución de consultas según esta causa fue la siguiente: 103.697 por ERAa (46,37\%), 68.604 por ERAb (30,68\%), 18.869 por EPOC $(8,44 \%)$, 13.591 por neumonía $(6,08 \%), 10.728$ por asma $(4,80 \%)$ y 8.128 por otras causas respiratorias $(3,63 \%)$. Los resultados generales, para todo el período, se presentan en la Tabla 2 .

\section{Tabla 2. Distribución de consultas de morbilidad de adultos en los 7 centros centinela de la provincia de Santiago de Chile, según diagnóstico, enero de 2003-diciembre de 2008}

\begin{tabular}{|lcc|}
\hline Diagnósticos & $\mathbf{n}$ & \% \\
CA & 1.170 .941 & 100 \\
CR & 223.617 & 19,10 \\
CNR & 947.324 & 80,90 \\
ERAa & 103.697 & $8,86(46,37)$ \\
ERAb & 68.604 & $5,86(30,68)$ \\
EPOC & 18.869 & $1,61(8,44)$ \\
Asma & 10.728 & $0,92(4,80)$ \\
Neumonía & 13.591 & $1,16(6,08)$ \\
OR & 8.128 & $0,69(3,63)$ \\
\hline
\end{tabular}

Fuente: Elaboración de los autores; n: Número de Consultas; $\%$ : Porcentaje de consultas respecto del total de Consultas de Adulto; CA: Consultas de morbilidad de adultos; CR: Consultas respiratorias; CNR: Consultas no respiratorias; ERAa: Enfermedad respiratoria aguda de vía aérea alta; ERAb: Enfermedad respiratoria aguda de vía aérea baja; EPOC: Enfermedad Pulmonar Obstructiva Crónica; OR: Otras causas respiratorias. Nota 1: Entre paréntesis se presenta el porcentaje de consultas por determinado diagnóstico, con respecto al total de consultas respiratorias, para cada año. 
$\mathrm{Al}$ agrupar los datos en promedios mensuales, se puede observar que las consultas respiratorias totales variaron entre un máximo de $36,17 \%$ en mayo de 2004 y un mínimo de 9,47\% en febrero de 2008, con respecto al total de CA. Al observar la variación estacional según diagnóstico específico, se observó que todas las enfermedades respiratorias alcanzaron su máximo en los meses de junio y julio, mientras la menor proporción de consultas se presentó entre diciembre y febrero.

En la Tabla 3 se muestran las proporciones de consultas por causa "respiratoria" y "no respiratoria", respecto del total de consultas de morbilidad de adultos, para cada año de la serie estudiada, así como el desglose de las respiratorias, por diagnóstico específico.

Con respecto a la variación anual de consultas en los mayores de 15 años, a lo largo de la serie 2003-2008 para cada enfermedad (Tabla 3), se observó que al aplicar un análisis de regresión en series de tiempo a los datos anuales, queda en evidencia una reducción significativa en el porcentaje de consultas por ERAa (reducción anual de $0,45 \%$; valor $\mathrm{p}=0,009$ ) y EPOC (reducción anual de $0,21 \%$; valor $\mathrm{p}=0,010$ ), al mismo tiempo que es posible observar una reducción no significativa en la proporción de consultas respiratorias en los mayores de 15 años (reducción anual de 0,78\%; valor $\mathrm{p}=0,089)$, en el porcentaje de consultas por ERAb (reducción anual de 0,16\%; valor $\mathrm{p}=0,300)$ y asma (reducción anual de $0,05 \%$; valor $\mathrm{p}=0,217)$.
Por otro lado, la proporción de consultas por neumonía experimentó un aumento no significativo (incremento anual de $0,07 \%$; valor $\mathrm{p}=0,395$ ).

\section{Resultados por grupo etario}

Para el período de 2003 al 2008, el volumen de CA según grupo etario, se distribuyó como sigue:

- Grupo de 15-44 años: 458.903 consultas, de las cuales $102.244(22,28 \%)$ fueron por causa respiratoria.

- Grupo de 45-64 años: 363.060 consultas, de las cuales $58.162(16,02 \%)$ fueron por causa respiratoria.

- Grupo de 65 años y más: 348.978 consultas, de las cuales $63.211(18,11 \%)$ fueron por causa respiratoria.

La proporción de diagnósticos para cada grupo etario se presenta en la Tabla 4.

$\mathrm{Al}$ comparar las diferencias entre las proporciones de consulta por determinado diagnóstico, según grupo etario, mediante la prueba de ANOVA de un factor y posterior contraste de Scheffé, queda en evidencia que la proporción de CR es significativamente mayor en el grupo de 15 a 44 años, a expensas de una proporción significativamente mayor de consultas por ERAa en este mismo grupo.

Por otro lado, la proporción de consultas por neumonía y EPOC fue significativamente mayor en el grupo de 65 y más años, ambos con un valor $\mathrm{p}=0,001$. La importancia relativa de las consultas

Tabla 3. Proporción de consultas en mayores de 15 años por diagnóstico, en centros Centinela de Atención Primaria, Provincia de Santiago, Chile, 2003-2008

\begin{tabular}{|cccccccccc|}
\hline Año & CA & CNR & CR & ERAa & ERAb & EPOC & Asma & Neumonía & OCR \\
2003 & 100 & 80,76 & 19,24 & $9,59(49,83)$ & $5,70(29,63)$ & $1,98(10,30)$ & $0,87(4,52)$ & $0,75(3,92)$ & $0,35(1,80)$ \\
2004 & 100 & 79,09 & 20,91 & $10,00(47,82)$ & $6,46(30,89)$ & $1,92(9,21)$ & $1,02(4,87)$ & $0,93(4,43)$ & $0,58(2,80)$ \\
2005 & 100 & 78,74 & 21,26 & $8,83(41,55)$ & $6,56(30,85)$ & $1,74(8,17)$ & $1,12(5,28)$ & $1,64(7,73)$ & $1,36(6,42)$ \\
2006 & 100 & 81,48 & 18,52 & $8,65(46,72)$ & $5,24(28,27)$ & $1,78(9,61)$ & $0,95(5,13)$ & $1,21(6,53)$ & $0,69(3,74)$ \\
2007 & 100 & 81,71 & 18,29 & $8,46(46,27)$ & $5,85(32,01)$ & $1,29(7,07)$ & $0,84(4,59)$ & $1,35(7,41)$ & $0,48(2,65)$ \\
2008 & 100 & 84,09 & 15,91 & $7,37(46,35)$ & $5,23(32,85)$ & $0,86(5,43)$ & $0,66(4,18)$ & $1,09(6,87)$ & $0,69(4,32)$ \\
\hline
\end{tabular}

Fuente: Elaboración de los autores. CA: Proporción de Consultas de adultos; CR: Proporción de Consultas respiratorias; CNR: Proporción de Consultas no respiratorias; ERAa: Proporción de consultas por Enfermedad respiratoria aguda de vía aérea alta; ERAb: Proporción de consultas por Enfermedad respiratoria aguda de vía aérea baja; EPOC: Proporción de consultas por Enfermedad Pulmonar Obstructiva Crónica; OCR: Proporción de consultas por Otras Causas Respiratorias. Nota 1: Entre paréntesis se presenta el porcentaje de consultas por determinado diagnóstico, con respecto al total de consultas respiratorias, para cada año. 
Tabla 4. Proporción de consultas respiratorias por diagnóstico específico para cada grupo etario en la Provincia de Santiago, Chile, 2003-2008

\begin{tabular}{|c|c|c|c|c|c|c|c|c|}
\hline \multirow[b]{3}{*}{ Diagnóstico } & \multicolumn{8}{|c|}{ Grupos de Edad (en años) } \\
\hline & \multicolumn{2}{|c|}{$15-44$} & \multicolumn{2}{|c|}{$45-64$} & \multicolumn{2}{|c|}{65 y más } & \multicolumn{2}{|c|}{15 y más } \\
\hline & $\mathbf{n}$ & $\%$ & $\mathbf{n}$ & $\%$ & $\mathbf{n}$ & $\%$ & n & $\%$ \\
\hline ERAa & 61.417 & 13,38 & 24.326 & 6,70 & 17.954 & 5,14 & 103.697 & 8,86 \\
\hline ERAb & 29.083 & 6,34 & 19.209 & 5,29 & 20.312 & 5,82 & 68.604 & 5,86 \\
\hline EPOC & 2.889 & 0,63 & 4.993 & 1,38 & 10.987 & 3,15 & 18.869 & 1,61 \\
\hline Asma & 3.258 & 0,71 & 3.459 & 0,95 & 4.011 & 1,15 & 10.728 & 0,92 \\
\hline Neumonía & 2.374 & 0,52 & 3.158 & 0,87 & 8.059 & 2,31 & 13.591 & 1,16 \\
\hline$C R$ & 102.244 & 22,28 & 58.162 & 16,02 & 63.211 & 18,11 & 223.617 & 19,1 \\
\hline CA & 458.903 & 100 & 363.060 & 100 & 348.978 & 100 & 1.170 .941 & 100 \\
\hline
\end{tabular}

Fuente: Elaboración de los autores; n: Número de Consultas; \%: Porcentaje de consultas respecto del total de Consultas para cada grupo etario; CA: Consultas de morbilidad de adultos; CR: Consultas respiratorias; ERAa: Enfermedad respiratoria aguda de vía aérea alta; ERAb: Enfermedad respiratoria aguda de vía aérea baja; EPOC: Enfermedad Pulmonar Obstructiva Crónica.

por neumonía, entre los diferentes grupos etarios, queda de manifiesto en la Figura 1.

\section{Canales endémicos}

Los resultados anteriores permiten expresar los datos en función de mínimos, medianas y máximos observados durante el período, con el objeto de construir canales endémicos, que puedan orientar al lector respecto de las características de un año normal de consultas.

Los canales endémicos para el grupo de mayores de 15 años, durante el período 2003-2008 se presentan en las Figuras 2 a 7. Adviértase que las escalas porcentuales en el eje de ordenadas varían para cada Figura.

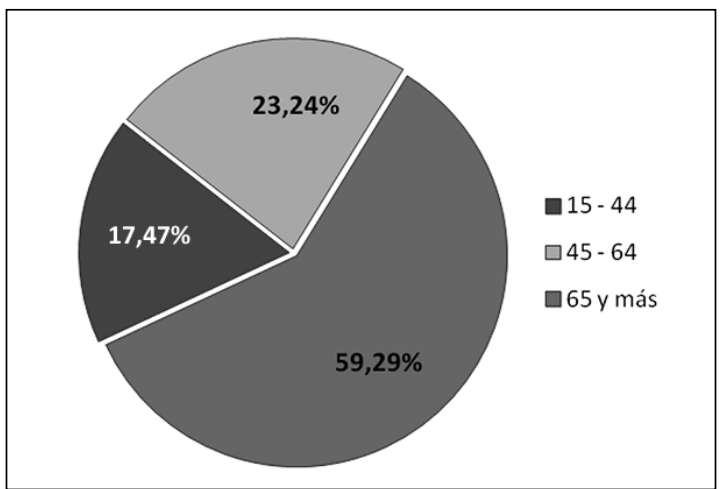

Figura 1. Distribución de consultas por neumonía entre cada grupo etario, provincia de Santiago, Chile, 2003-2008. Fuente: Elaboración de los autores.

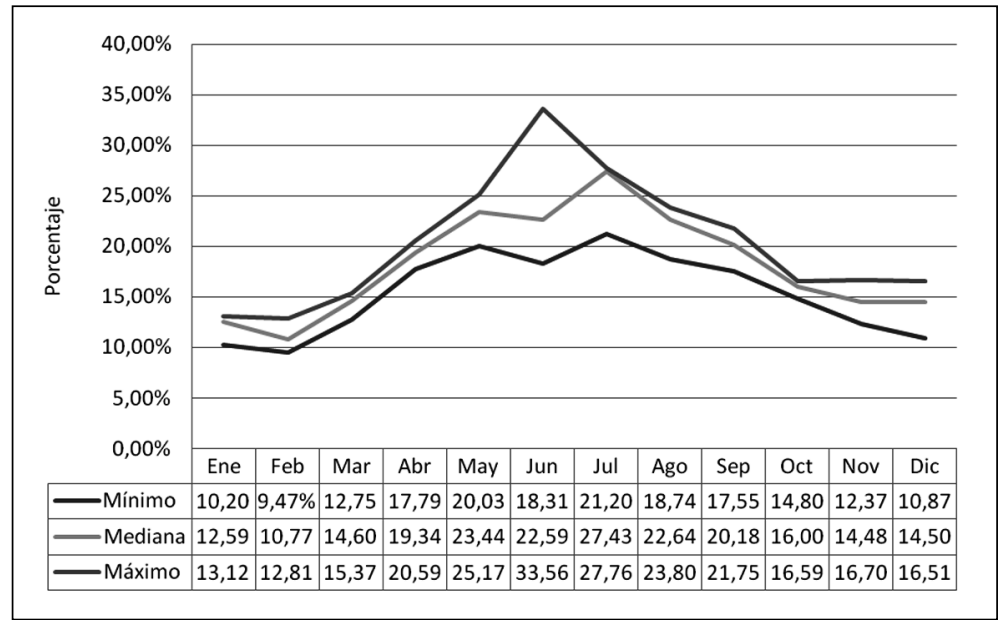

Figura 2. Proporción de consultas respiratorias en mayores de 15 años, con respecto al total de Consultas de Adulto, Provincia de Santiago, Chile, 2003-2008. 
Consultas respiratoria de adultos en Santiago - C. Olmos et al
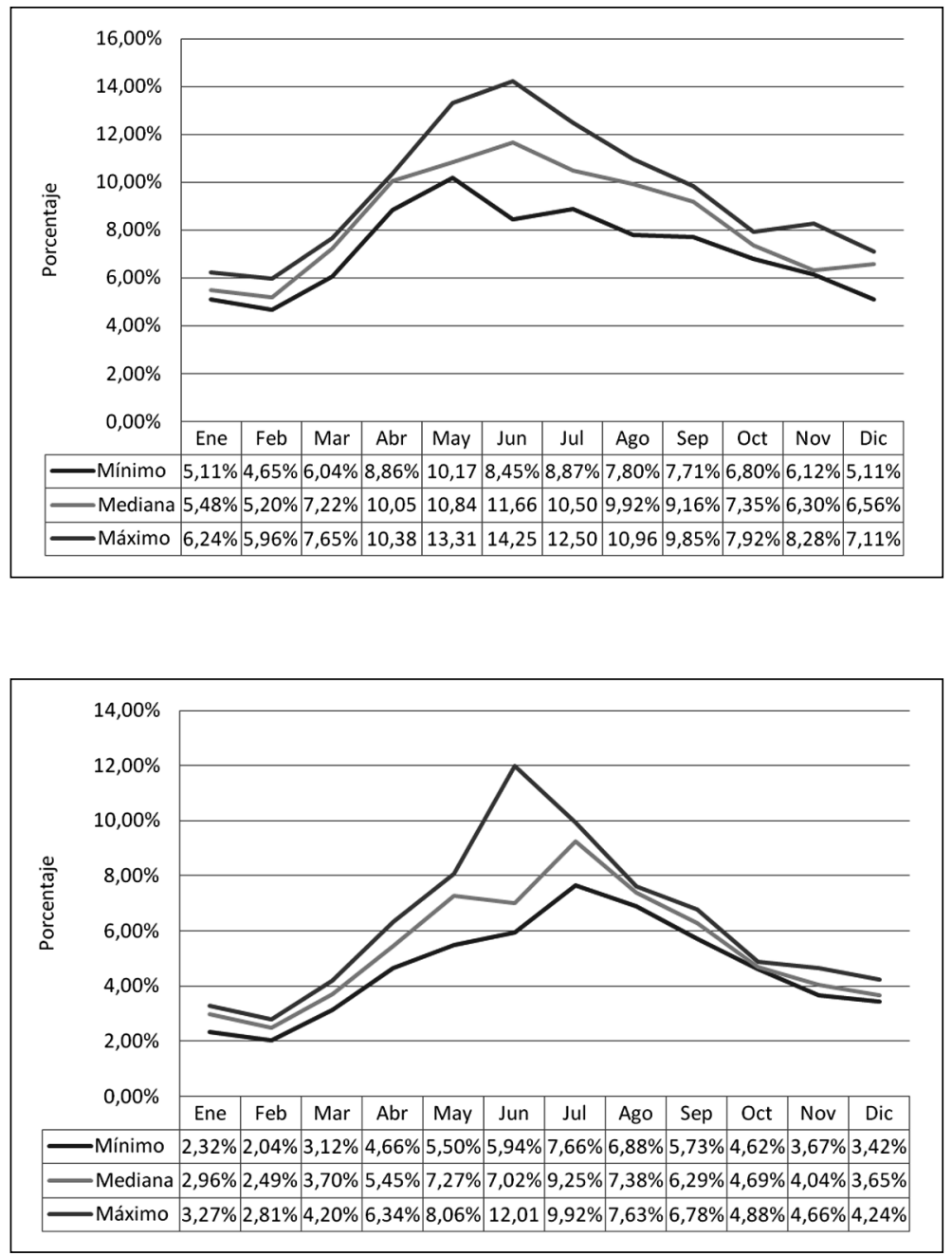

Figura 3. Proporción de consultas por ERAa en mayores de 15 años, con respecto al total de Consultas de Adulto, Provincia de Santiago, Chile, 2003-2008.
Figura 4. Proporción de consultas por ERAb en mayores de 15 años, con respecto al total de Consultas de Adulto, Provincia de Santiago, Chile, 2003-2008.

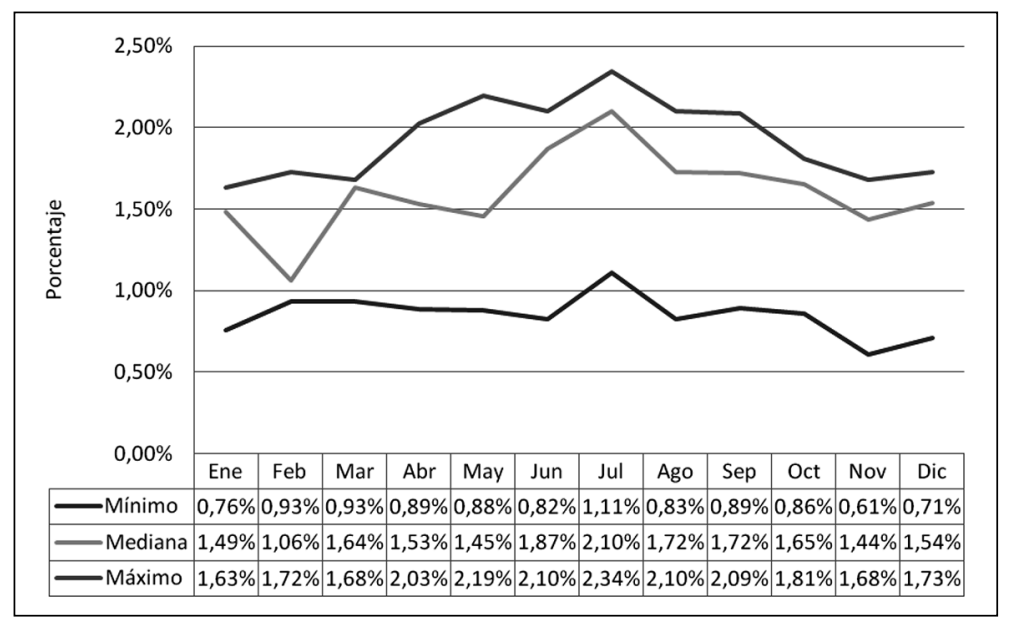

Figura 5. Proporción de Consultas por EPOC en mayores de 15 años, con respecto al total de Consultas de Adulto, Provincia de Santiago, Chile, 2003-2008. 

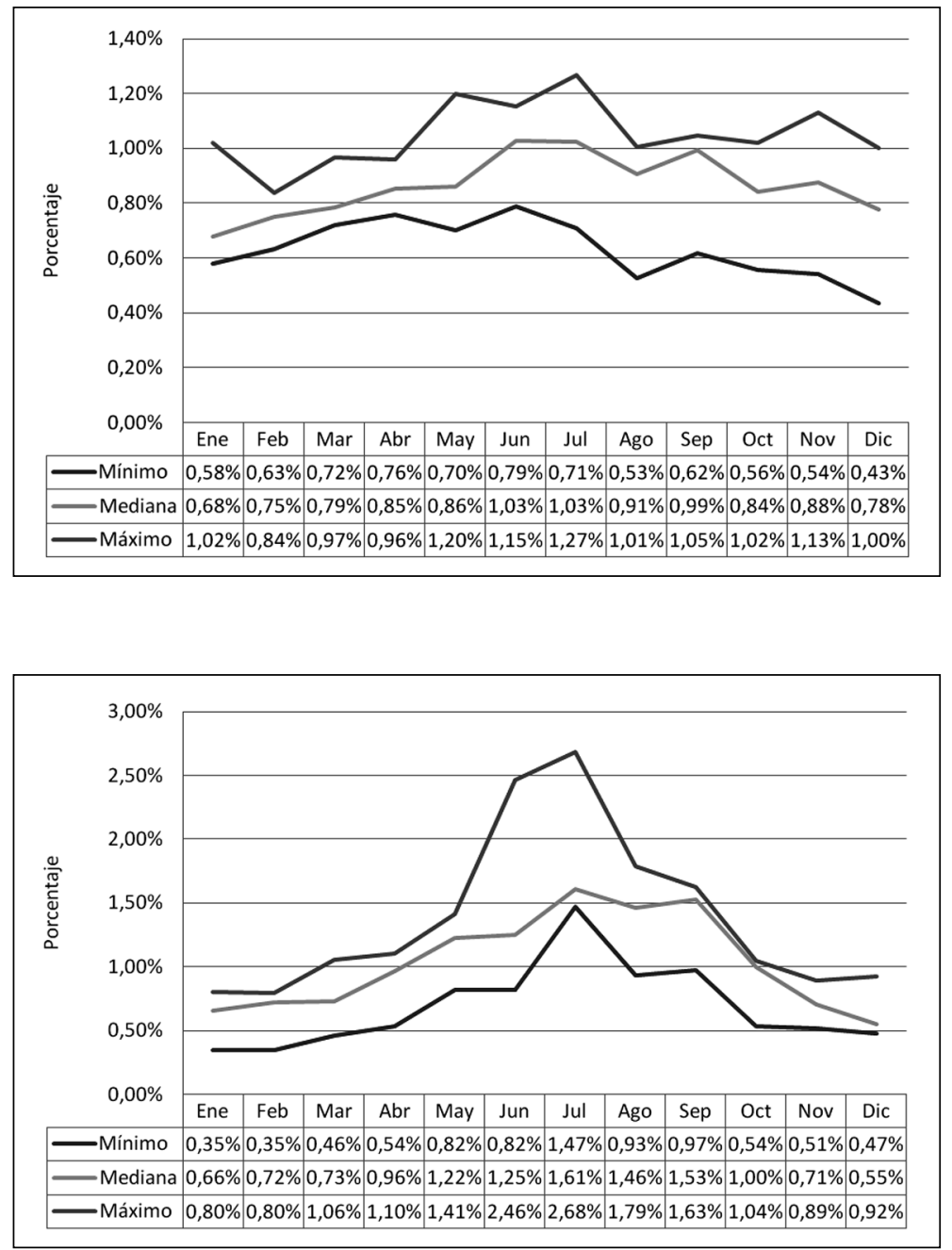

Figura 6. Proporción de Consultas por Asma en mayores de 15 años, con respecto al total de Consultas de Adulto, Provincia de Santiago, Chile, 2003-2008.
Figura 7. Proporción de Consultas por Neumonía en mayores de 15 años, con respecto al total de Consultas de Adulto, Provincia de Santiago, Chile, 2003-2008.

\section{Discusión}

Los centros de salud seleccionados permitieron observar a 5,9\% del total de la población de la provincia de Santiago ${ }^{15}$, cifra sin precedentes en este tipo de estudios.

En relación a los diagnósticos, resulta necesario destacar, que si bien están basados en parámetros preferentemente clínicos, la calidad y veracidad de la información está respaldada por el hecho de que todos fueron realizados por médicos y contaron con la presencia de kinesiólogo capacitado y familiarizado con la nomenclatura usada en enfermedades respiratorias, el uso de formularios estandarizados, el control de la calidad de los registros, la asesoría técnica permanente de los investigadores al equipo local y las visitas a terreno.

$\mathrm{Al}$ analizar los resultados generales, el presente trabajo revela que las enfermedades respiratorias, constituyen un quinto de todas las consultas de morbilidad, superando los valores mostrados en investigaciones nacionales previas para los mismos grupos etarios $^{3-9}$.

Asimismo, se observa una leve reducción en el porcentaje de consultas por causa respiratoria, por ERAa, ERAb y EPOC, mientras que la proporción de consultas por asma y neumonía, prácticamente se mantienen sin variaciones, a excepción del grupo de mayores de 65 años, en que la proporción de consultas por neumonía aumentó. 
Uno de los aspectos a considerar para entender aquello, es el desarrollo del Programa ERA a partir del año 2001 como piloto, y 2002-2003 en todo el territorio nacional, hasta la actualidad. En ese contexto, y como un símil del exitoso Programa IRA de $1990^{17}$, se implementaron estrategias de prevención, promoción y manejo clínico precoz, oportuno y sistemático de las patologías respiratorias agudas y crónicas del adulto, uso de normas técnicas protocolizadas y disponibilidad completa de fármacos. Todo esto permitió aumentar la capacidad diagnóstica, educar a la población, fortalecer la adherencia al tratamiento y ampliar la cobertura de patologías como la neumonía.

Con respecto a los resultados por edad, se muestra que para todos los grupos etarios, la mayor proporción de consultas respiratorias pertenece a consultas por ERAa, seguido de las consultas por ERAb, sin embargo, la composición interna de las consultas por diagnóstico específico para cada grupo etario es dispar.

La mayor proporción de consultas por ERAa y de ERAb pertenecen al grupo de 15 a 44 años, mientras que las consultas por EPOC y neumonía se hacen notar en los mayores de 65 años. Esto quiere decir que los adultos jóvenes sufren mayoritariamente de patologías agudas de menor gravedad, mientras que los adultos mayores padecen de enfermedades crónicas y agudas graves. La proporción de consultas por asma prevalece de igual manera en los tres grupos etarios, confirmando que es una entidad patológica presente a lo largo del ciclo vital.

Se observa en todos los grupos etarios, una marcada estacionalidad respecto de las consultas respiratorias en general, consultas por ERAa, ERAb y neumonía, en relación directa con factores de riesgo como los altos niveles de contaminación ${ }^{6}$ y circulación de virus durante los meses de frío ${ }^{5}$. En contraste, las consultas por EPOC y asma se mantienen presentes durante todos los meses del año, acorde a sus patrones de cronicidad.

Una de las posibles limitaciones de este estudio es que, tal como ha sido descrito, en la metodología de registros utilizada se consigna el número de consultas y no el número de episodios de enfermedad, y, por lo tanto, no se puede calcular incidencia. Esto debiera ser más relevante en el análisis de consultas por neumonía, en que un paciente podría haber consultado más de una vez por episodio, por tratarse de un cuadro más grave, o por la cronicidad de patologías como EPOC y asma. En síntesis, se confirma que las enfermedades respiratorias son un grupo importante de causa de consultas en los adultos y que nuestros adultos mayores son quienes más padecen las consecuencias negativas de estas patologías.

Por otro lado, se hace evidente la importancia de contar con un sistema de vigilancia y las bondades que otorga esta modalidad de recolección y análisis de la información.

Por la importancia que tienen las consultas respiratorias en los sistemas de atención de Chile $y$ en virtud de los resultados generales, se hace indispensable que las autoridades del sector público y privado, dispongan de esta información, a partir de la cual puedan asignar o redestinar recursos en programas de promoción, educación, prevención $\mathrm{y}$ tratamiento de estas enfermedades.

\section{Referencias}

1. Departamento de Estadísticas e Informaciones-Ministerio de Salud-Chile. Distribución porcentual de las defunciones por grandes grupos de causas de muerte. Chile 1960, 1970, 1980, 1990, 2000 y 2009. Disponible en: http://www.deis.cl/estadisticas-mortalidad/

2. Departamento de Estadísticas e InformacionesMinisterio de Salud-Chile. Distribución. Estadísticas por Egresos Hospitalarios. Chile 2006, 2007, 2008 y 2009. Disponible en: http://www.deis.cl/estadisticasegresoshospitalarios/ Acceso el 07 de mayo de 2014.

3. Valdivia G. Epidemiología de la neumonía del adulto adquirida en la comunidad. Rev Chil Infectol 2005; 22 (1): 11-7.

4. Dintrans K, Andrade C, Sánchez J, Mendoza J. Neumonía adquirida en la comunidad en adultos, en el curso de la campaña de invierno 2003 en el Hospital San Juan de Dios. Rev Chil Enferm Resp 2005; 21 (1): 15-22.

5. Rebagliati R, Serri M, Perret C, Guzmán A, Azócar T, Ferres M, et al. Perfil clínico-epidemiológico de las infecciones por virus respiratorios en adultos hospitalizados durante la estación de Influenza 2004. Rev Chil Infectol 2006; 23 (2): 111-7.

6. Prieto M, Mancilla P, Astudillo P, Reyes A, Román O. Exceso de morbilidad en niños y adultos mayores en una comuna de Santiago con alta contaminación atmosférica por partículas. Rev Med Chile 2007; 135: 221-8.

7. Gil R, Undurraga A, Saldías F, Jiménez P, Barros M. Estudio multicéntrico de factores pronósticos en adultos hospitalizados por neumonía adquirida en la comunidad. Rev Med Chile 2006; 134: 1357-66. 
8. Medina E, Kaempffer A, Cornejo E, Hernández E. Características de la atención brindada en un establecimiento de atención primaria. Rev Chil Salud Pública 1998; 2 (3): 180-84.

9. Cornejo L, Figueroa P, Romero P, Chaparro L, Cortés L, López I, et al. Presencia de sintomáticos respiratorios adultos en la consulta por morbilidad general: Consultorio Renca 1983. Bol Hosp San Juan de Dios 1985; 32 (6): 388-92.

10. Mazzei M. Envejecimiento poblacional en Chile y América Latina. Rev Chil Salud Pública 2003; 7: 104-5.

11. Ministerio de Salud Chile. Norma Técnica "Programa de Control de las Enfermedades Respiratorias del Adulto en Chile”. Resolución exenta Nº 2282 año 2002.

12. Astudillo P, Mancilla P, Olmos C, Reyes A. Epidemiología de las consultas pediátricas respiratorias en Santiago de Chile desde 1993 a 2009. Rev Panam Salud Pública 2012; 32 (1): 56-61.

13. Organización Mundial de la Salud. "Clasificación estadística internacional de enfermedades y problemas relacionados con la salud; décima revisión (CIE-10), Cap X: Enfermedades del sistema respiratorio. 1995.

14. Lazcano F, Westhof D, Lima J. Sitios centinelas, monitoreo y evaluación. UNICEF CA. 1991. Mimeo p 1- 8.

15. Instituto Nacional de Estadísticas. Censo de Población y Vivienda 2002. Chile. Disponible en: http://www.ine. cl/canales/chile_estadistico/censos_poblacion_vivienda/ censo_pobl_vivi.php Acceso el 07 de mayo de 2014.

16. Aranda, Romero H. Topoclimatología de la cuenca de Santiago y sus efectos en la contaminación atmosférica y en la salud. Enf Resp Cir Tor 1989; 5: 24-30.

17. Girardi G, Astudillo P, Zúñiga F. “El Programa IRA en Chile: Hitos e Historia". Rev Chil Pediatr 2001; 72 (4): 292-300

18. Ministerio de Salud de Chile, Fondo Nacional de Salud. Boletín Estadístico 2011-2012. Chile. Disponible en: http://www.fonasa.cl/wps/wcm/connect/ Internet/SA-General/Informacion+Corporativa/ Estadisticas+Institucionales/ Acceso el 07 de mayo de 2014. 